\title{
A dynamic bipolar electrode array for visualized screening of electrode materials in light-emitting electrochemical cells
}

\author{
Shiyu Hu and Jun Gao* \\ Department of Physics, Engineering Physics and Astronomy, Queen's University, Kingston, Ontario, K7L 3N6, Canada
}

\begin{abstract}
Charge injection at a metal/semiconductor interface is of paramount importance for many chemical and physical processes. The dual injection of electrons and holes, for example, is necessary for electroluminescence in organic light-emitting devices. In an electrochemical cell, charge transfer across the electrode interface is responsible for redox reactions and faradic current flow. In this work, we use polymer light-emitting electrochemical cells (PLECs) to visually assess the ability of metals to inject electronic charges into a luminescent polymer. Silver, aluminum or gold micro-discs are deposited between the two driving electrodes of the PLEC in the form of a horizontal array. When the PLEC is polarized, the individual discs functioned as bipolar electrodes (BPEs) to induce redox p- and n-doping reactions at their extremities, which are visualized as strongly photoluminescence-quenched growth in the luminescence polymer. The three metals initially generate highly distinct doping patterns that are consistent with differences in their work function. Over time, the doped regions continue to grow in size. Quantitative analysis of the $n / p$ area ratio reveals an amazing convergence to a single value for all 39 BPEs, regardless of their metal type and large variation in the size of individual doped areas. We introduce the concept of a dynamic BPE, which transforms from an initial metal disc of fixed size to one that is a composite of $\mathrm{p}$ - and n-doped polymer joined by the initial metallic BPE. The internal structure of the dynamic BPE, as measured by the $\mathrm{n} / \mathrm{p}$ area ratio, reflects only the properties of the mixed conductor of the PLEC active layer itself when the area ratio converges.
\end{abstract}

Since their inception in 1995, light-emitting electrochemical cells (LECs) have been actively researched for the interesting science of luminescent mixed conductors and for their potential applications as low-cost display and lighting devices.1-12 The original LECs contained a solid polymer electrolyte (SPE) mixed with a luminescent conjugated polymer (CP). One can construct such a polymer LEC (PLEC) to generate electroluminescence (EL) by contacting the active layer with two driving electrodes (DEs) without express concern for their work function or the separation.13-14 The simple structure of PLECs, however, belies a complex operating mechanism that involves redox reactions, ion redistribution, electronic charge transport and junction EL. This is true for LECs made from CPs, organic small molecules, and ionic transition metal complexes. 15-20 The reduction or oxidation of the LEC light emitter constitutes an electrochemical $n$ - or p-doping process when counterions from the electrolyte compensate the injected electronic charges. The doping process distinguishes an LEC from an organic light-emitting diode that does not contain mobile ions.

Recently, PLECs have emerged as a new, solid-state platform to study bipolar electrochemistry (BPEC),21 a diverse and resurgent field of research with many unique applications.22-32 In BPEC, the reactions of interest occur at an electrically floating bipolar electrode (BPE).33-35 In a PLEC, one or multiple BPEs can be formed on top of, beneath, or within the active layer via vacuum deposition, ink-jet printing, dispersion and many other techniques.
The presence of a BPE drastically alters the doping patterns, conductivity and cell current of a PLEC.36-38 Many of the changes caused by the BPEs can be easily visualized and corroborated via photoluminescence (PL) and $p-n$ junction EL imaging.

PLECs allowed for the vivid illustration of the BPEC principles via time-lapsed imaging. Moreover, the solidstate nature of a PLEC led to novel BPE types such as serial-connected $\mathrm{p}-\mathrm{n}$ junctions,36, $38 \mathrm{p}$ - and n-doped regions of the same PLEC layer, and a laser illuminated regions of the PLEC film that did not initially contain any BPE.37, 39 BPEC research typically employs a liquid electrolyte in which the BPE is immersed. PLECs provides a simple and yet powerful platform to conduct BPEC research in solid state. In return, the inclusion of BPEs gave rise to novel junction structures such as the "bulk homojunction" that displayed vastly improved EL output and a giant opencircuit voltage.40-42

In this paper, we present a proof-of-concept demonstration of a visualized screening platform for electrode materials based on a PLEC. The metals of interest are fabricated as horizontal BPE arrays. The doping patterns emerged from the BPEs are quantitatively analyzed as a function of metal type, testing temperature and time elapsed. The PLEC of this study has a planar or lateral (vs. sandwich) configuration with an $11 \mathrm{~mm}$ separation between the DEs, as shown in Figure 1(a). Thirteen metal discs made of aluminum (Al), silver (Ag) or gold $(\mathrm{Au})$, are deposited in-between the Al DEs to form a horizontal array. The various dimensions are labelled on 
the images. The images were taken under UV illumination to reveal the strong PL of the luminescent CP. The inset of Figure 1(a) is a full view of the PLEC under ambient light.

BPE arrays are frequently employed in sensing and screening applications within a conventional liquid electrochemical cell that contains the analyte species. The use of an array, as opposed to a single BPE allows for statistical analysis of data from multiple reaction sites. When the BPEs are differently functionalized, it provides an efficient platform for multiplexed, parallel detection different materials such as catalysts or biomolecules. 43-55 The reporting mechanism of the BPE reactions is typically electrochemiluminescence.

In the current study, the 13 discs of each BPE array are identical in size and metal. Figure 1(b) shows the PL image of a PLEC $15 \mathrm{~s}$ after a $200 \mathrm{~V}$ bias was applied to the Al DEs. The BPEs are made of evaporated silver. At this moment, four different doping reactions are highly visible due to the doping reactions' strong quenching of PL. Notably, all 13 discs displayed p- and n-doping in their opposite poles, conforming their functions as individual BPEs. The BPE doping resembles the shape of a dumbbell or molecular $p$ orbital. The individual areas of the BPE p- and n-doped regions are calculated in Matlab as a measure of BPE doping progression. Although Figure 1 (b) displays an evenly matched BPE $\mathrm{p}$ - and n-doping, detailed analysis of the doping progression reveals such is not the case for all stages of operation. Significant variations in doping area and area ratio in early stages allowed for easy differentiation of three BPE metals. At a later stage, however, the $n / p$ area ratio converges to a single value, regardless of the metal type.

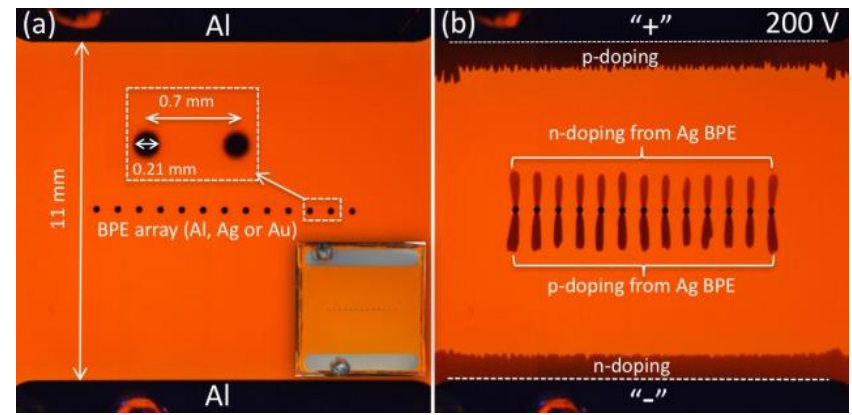

Figure 1. (a) Image of a planar PLEC under UV illumination showing red PL of the polymer layer. The Al DEs and a Ag BPE array appear in black. The various dimensions are denoted on the images. The inset shows the PLEC under room light. Two indium balls attached to the end of gold-coated tungsten probes make contact to the DEs. (b) PL image of the PLEC $15 \mathrm{~s}$ after a bias voltage of $200 \mathrm{~V}$ was applied to the DEs with polarity shown. The white dashed lines denote the edges of the DEs. The various doped regions are labelled.

\section{EXPERIMENTAL DETAILS}

The PLECs were fabricated on the back side of $16 \mathrm{~mm}$ by $16 \mathrm{~mm}$ ITO-coated glass substrates from a casting solution containing three materials: a luminescent CP, a SPE polymer and a salt. The luminescent $\mathrm{CP}$ was poly[2methoxy-5-(2-ethylhexyloxy)-1,4-phenylenevinylene], or MEH-PPV, was synthesized by OLEDKing Optoelectronic
Materials Ltd, China with a molecular weight (Mw) of $3.3 \times$ 105 and a polydispersity index of 1.4 . The SPE polymer was polyethylene oxide (PEO, $\mathrm{Mw}=2 \mathrm{M}$ ) and the salt was potassium trifluoromethanesulfonate (KTf). Both PEO and KTf were purchased from Aldrich and used as received. Cyclohexanone solutions of MEH-PPV (10 mg/mL) and PEO+KTf (20 mg+3.85 mg/mL) were prepared separately and mixed to create a casting solution containing $\mathrm{MEH}-$ PPV, PEO, and KTf with a weight ratio of 1:1.3:0.25. The casting solution was stirred on a magnetic hotplate at 25 ${ }^{\circ} \mathrm{C}$ for at least 5 hours before use. For each film, $100 \mu \mathrm{L}$ of the solution was dispensed onto a glass substrate, followed by spinning at $2000 \mathrm{rpm}$ and drying at $50{ }^{\circ} \mathrm{C}$ for 5 hours to remove any residual solvent. The thickness of resulting polymer film was $392 \pm 4 \mathrm{~nm}$, as measured with a DektakXT stylus profiler. A pair aluminum DEs $(100 \mathrm{~nm})$, separated by $11 \mathrm{~mm}$, were thermally evaporated onto the polymer film through a shadow mask. To complete the devices, an array of $\mathrm{Al}(100 \mathrm{~nm}), \mathrm{Ag}(50 \mathrm{~nm})$ or $\mathrm{Au}(30 \mathrm{~nm})$ discs were thermally evaporated through a shadow mask onto the devices. Each device has thirteen identical BPE disks, located near the centers of the devices, as shown in Figure 1(a). The above device processing steps were carried out in a MBraun integrated glove box/evaporator system filled with dry nitrogen.

The finished devices were transferred into a Janis ST500 micro-manipulated cryogenic probe station for testing under vacuum $(\sim 5 \times 10-4$ Torr $)$. A layer of thermoconductive paste was applied between the goldcoated probe station stage and the substrate. The device was illuminated by a $365 \mathrm{~nm}$ UV ring lamp rested on the quartz window of the chamber. A LabView controlled Keithley 237 source measurement unit was used to apply a $200 \mathrm{~V}$ bias to the driving electrodes and simultaneously measure the cell current. The devices were heated and maintained at temperatures of either $340 \mathrm{~K}$ or $360 \mathrm{~K}$. The PL images were captured with a computer-controlled Nikon D300 digital SLR camera equipped with a Tamron $90 \mathrm{~mm}$ 1:1 macro-lens. The captured images were eventually processed by Matlab R2017b to extract doping propagation data.

\section{RESULTS}

Figure 2 is a composite graph showing the doping patterns of all three BPE arrays. In this Figure as well as all other Figures, the elapsed time was measured relative to the moment a $200 \mathrm{~V}$ bias was applied to the PLEC. For each BPE array, images at $\mathrm{t}=3 \mathrm{~s}$ and $6 \mathrm{~s}$ are cropped to a size of $12 \mathrm{~mm}$ by $2 \mathrm{~mm}$ centered around the BPE and stitched together. For all the images shown, the doping above the BPE discs are n-doping. The PLECs containing these BPE arrays are identical and tested under the same conditions.

First, we examine the Ag array, as shown in Figure 2(b). Unlike the image of Figure 1(b), which is taken from the same PLEC at $t=15 \mathrm{~s}$, these early images show that the BPE $\mathrm{p}$ - and n-doping differ greatly in size (area). At $\mathrm{t}=3 \mathrm{~s}$, only $\mathrm{n}$-doping is visible atop the Ag discs. At $\mathrm{t}=6 \mathrm{~s}$, some BPE $\mathrm{p}$ doping is visible below the BPEs, but is only $1 / 6$ the size of n-doping on average. The prompt and dominant occurrence of n-doping suggests two criteria have been 
met-the injection of electrons from the cathodic pole of $\mathrm{Ag}$ BPEs that causes the reduction of MEH-PPV, and the insertion of $\mathrm{K}_{+}$counterions near the reduced sites. The much weaker p-doping, on the other hand, suggests the injection of holes and/or the insertion of $\mathrm{CF}_{3} \mathrm{SO}_{3}$ - counterions are insufficient. Before the polymer is doped, electron or hole injection is strongly dependent on the injection barrier and therefore the work function of the metal BPE. The availability of counter-ions and their transport, on the contrary, is a bulk property of the PLEC film.

The doping images shown in Figure 2(c) clearly point to metal work function, as opposed to the polymer film itself, as the main cause of the asymmetry in BPE p- and ndoping. Contrary to the observation of Figure 2(b), the initial doping from the Au BPE array is mainly p-doping, as shown in the $t=3 \mathrm{~s}$ image. At $\mathrm{t}=6 \mathrm{~s}$, the average size of BPE p-doping from the Au array is 18 times larger than the $\mathrm{Ag}$ array. This observation can be easily explained by Au's much higher work function $(4.4 \pm 0.1) \mathrm{eV}$ compared to Ag's work function of $(3.7 \pm 0.1) \mathrm{eV} .56 \mathrm{~A}$ high work function metal facilitates hole injection (oxidation) which is a necessary condition for p-doping. The importance of metal work function for charge injection in polymer light-emitting diodes is well established.57

The work function explanation of the doping asymmetry is further supported by the observations of Figure 2(a). Since $\mathrm{Al}(3.4 \pm 0.1) \mathrm{eV}$ has a similar work function as $\mathrm{Ag}$, the doping from the Al BPEs is also predominately n-doping, especially at $t=3 \mathrm{~s}$. The results of Figure 2 are repeatable. Three additional PLECs were fabricated, each containing a $\mathrm{BPE}$ array of $\mathrm{Al}, \mathrm{Ag}$ or $\mathrm{Au}$ and tested at $340 \mathrm{~K}$. The lower testing temperature slowed down the doping progression, as seen in Figure S1 (Supplemental Information). The same asymmetric doping patterns have been observed in all three devices. At $t=3 \mathrm{~s}$, the Au BPE only showed p-doping while the Al BPE only showed n-doping. The $t=6 \mathrm{~s}$ images are also consistent with the trend of Figure 2. The highly distinct doping patterns of Figure 2 and Figure S1 thus offer visual evidence to the metal's ability for electron or hole injection which is related to its work function.
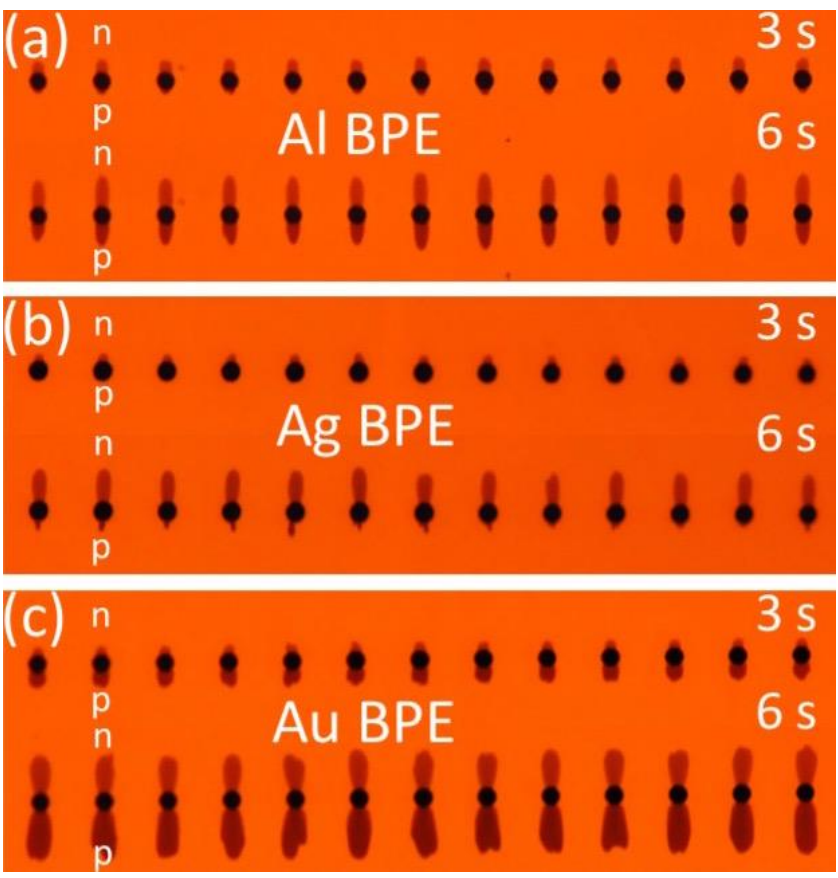

Figure 2. Composite PL graph showing the doping patterns of three BPE arrays at two different times. The three PLECs with the $\mathrm{Al}, \mathrm{Ag}$ and $\mathrm{Au} \mathrm{BPE}$ arrays are otherwise identical and tested under the same bias voltage of $200 \mathrm{~V}$ bias and a temperature of $360 \mathrm{~K}$. For each BPE array, images at $t=3 \mathrm{~s}$ and $6 \mathrm{~s}$ are cropped to a size of $12 \mathrm{~mm}$ by $2 \mathrm{~mm}$ centered around the BPE and stitched together. The letters " $p$ " and " $n$ " denote the BPE doping type.

We note that in Figure 2, significant $\mathrm{n}$-doping is also present atop the Au BPEs at $\mathrm{t}=6 \mathrm{~s}$. This is not an indication that $\mathrm{Au}$ is also a good electron injector. Rather, it is the consequence of doping propagation. The implication of this phenomenon will be discussed later. The continued doping propagation is shown quantitatively in Figure 3. The p- and n-doped areas, averaged over all thirteen BPEs for each BPE array, are plotted against time and shown for all six PLECs tested. The doped areas are calculated from timelapsed images such as those shown in Figure 1(b), Figure 2 and Figure S1. The error bars represent one standard deviation of the mean. 
(a)

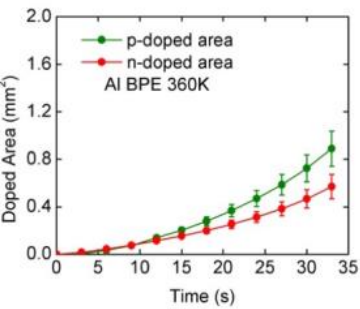

(b)

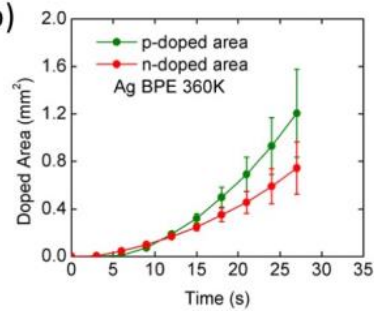

(c)

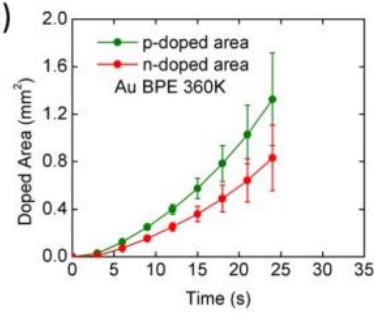

(d)

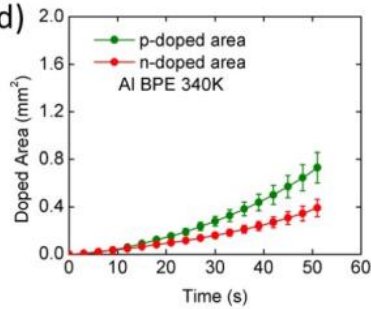

(e)

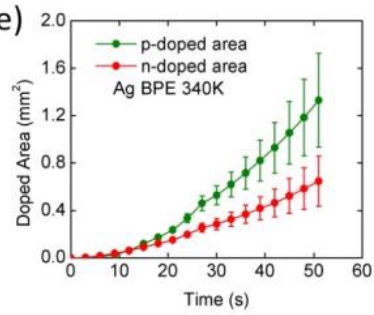

(f)

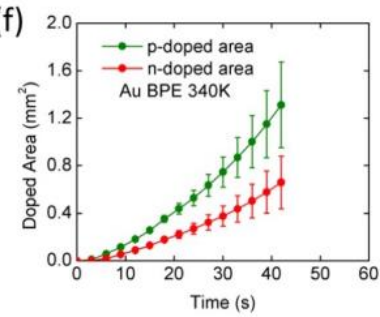

Figure 3. Average BPE p- and n-doped area vs. elapsed time for six PLECs, each containing a BPE array of $\mathrm{Al}, \mathrm{Ag}$, or $\mathrm{Au}$. The PLECs were biased at $200 \mathrm{~V}$ and tested at either $360 \mathrm{~K}(\mathrm{a}-\mathrm{c})$ column) or $340 \mathrm{~K}$ (d-f). The doped areas were extracted with a Matlab script on PL images showing the BPE doping before their contact with any doping originated from the driving electrode. The BPE type and test temperature are indicated in the individual plots labelled (a)-(f).

In Figure 3, each row compares the same BPE type tested at $360 \mathrm{~K}$ vs. $340 \mathrm{~K}$. It is apparent that a higher testing temperature resulted in a faster expansion of BPE doping areas for all three BPE types. At $340 \mathrm{~K}$, the slower doping progression allowed for more image frames to be used for the calculation before any encounter with the DE doping had occurred. In Figure 3, each column compares different BPE arrays tested at the same temperature. At both $360 \mathrm{~K}$ and $340 \mathrm{~K}$, the Au BPE array generated the fasted doping expansion, followed by the Ag and Al BPE arrays, respectively. The doping area vs. time curves exhibit significant curvature, signifying an accelerated growth of the doped regions in size.

As doping progresses, the spread in doped area increases, as indicated by the standard deviation in absolute terms. The large spread in doped area is visualized in Figure 4 for Ag BPEs tested at both $360 \mathrm{~K}$ and $340 \mathrm{~K}$. The image of Figure 4(a) was captured at $t=27 \mathrm{~s}$ for the PLEC tested at $360 \mathrm{~K}$. We see that the BPE p- and ndoping have grown to enormous sizes relative to that of the Ag BPE. The areas of BPE p-doping, n-doping as well as the area ratios are calculated for each of the thirteen BPEs and shown in Figure 4(b). The BPE doped areas of BPE\#1 and BPE\#9 differed by more than $100 \%$. Strikingly, the $\mathrm{n} / \mathrm{p}$ area ratios are nearly identical of $0.65 \pm 0.01$ for all

thirteen BPEs. The exact same behavior has been observed for the Ag BPE cell tested at $340 \mathrm{~K}$, as shown in Figure 4(c) and Figure 4(d). The only difference is a smaller $n / p$ area ratio at $0.49 \pm 0.01$. Additional images are analyzed in Figure S2 that also included images from the $\mathrm{Al}$ and $\mathrm{Au}$ BPEs. All display the same behavior. In the 2:1 scaled plots of calculated doped areas, the BPE p- and n-doped areas are nearly mirror-symmetric about zero for the $340 \mathrm{~K}$ data due to an area ratio that is close to 0.5 .
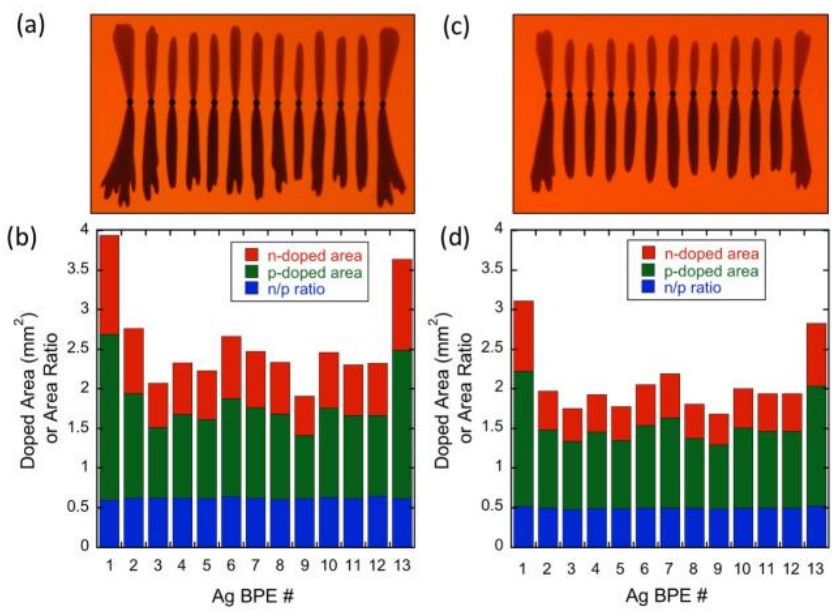

Figure 4. Doping patterns of Ag BPE arrays. (a) A PLEC with a Ag BPE array tested at $360 \mathrm{~K}$. The image was taken $27 \mathrm{~s}$ after a $200 \mathrm{~V}$ bias was applied to the driving electrodes of the PLEC. (b) Stacked bar diagram showing doped areas and area ratios for the image shown in (a); (c) A PLEC with a Ag BPE array tested at $360 \mathrm{~K}$. The image was taken $45 \mathrm{~s}$ after a $200 \mathrm{~V}$ bias was applied to the driving electrodes of the PLEC. (d) Stacked bar diagram showing doped areas and area ratios for the image shown in (c).

In Figure 5, the $n / p$ area ratio is plotted against time for all six PLECs tested. The area ratios are averages calculated from all thirteen BPEs in consecutive image frames taken at the indicated time. The error bar represents one standard deviation of the mean. The initial $(t<9 s)$ area ratios have a large spread. This reflects the highly distinct and asymmetric doping patterns such as the ones shown in Figure 2 and Figure S1. For both $\mathrm{Al}$ and Ag BPEs, the initial $\mathrm{n} / \mathrm{p}$ area ratios were higher than 1 due to a faster $\mathrm{n}$-doping growth from these BPEs. For the Au BPEs, the initial $n / p$ area rations are less than 0.5 as the BPE doping is predominately $\mathrm{p}$-type as favored by the high work function $\mathrm{Au}$.

The data in Figure 5 reveal a striking convergence of $n / p$ area ratio toward a constant. For PLECs tested at $360 \mathrm{~K}$, as shown in Figure 5(a), the converged area ratios are $0.64 \pm 0.02,0.62 \pm 0.01$, and $0.62 \pm 0.02$, respectively, for $\mathrm{Al}$, $\mathrm{Ag}$ and $\mathrm{Au}$ BPEs. For PLECs tested at $340 \mathrm{~K}$, as shown in Figure 5(b), the corresponding area ratios are $0.54 \pm 0.02$, $0.48 \pm 0.01$, and $0.50 \pm 0.03$, respectively. It is also observed that the area ratios barely changed in the last five frames for both temperatures. 

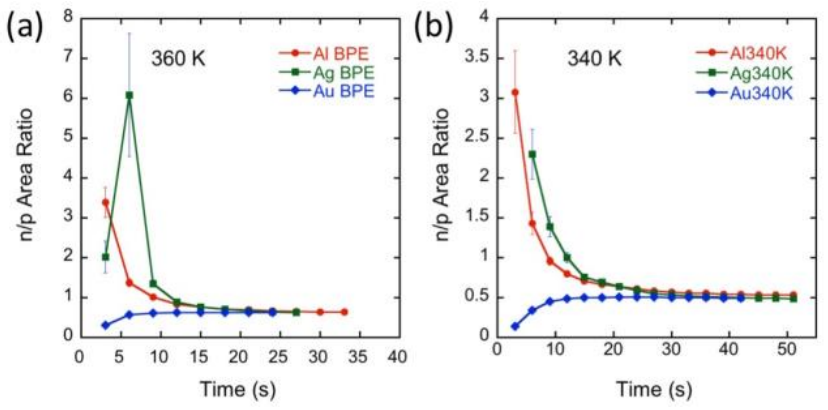

Figure 5. Calculated $n / p$ area ratios as a function of time for six PLECs. Each data point in these graphs represent the average $n / p$ area ratio among 13 BPEs calculated from a single frame of image taken at the indicated time. (a) For PLECs tested at $360 \mathrm{~K}$. (b) for PLECs tested at $340 \mathrm{~K}$.

\section{DISCUSSION}

We showed that BPE arrays, formed in the middle of extremely large PLECs, can induce electrochemical $p$ - and $\mathrm{n}$-doping at the extremities of the individual BPEs when the PLEC is polarized. The initial BPE is strongly affected by the work functions of the BPE metal, as shown in Figure 2 and Figure S1. A Au-BPE array strongly favors p-doping, while $\mathrm{Al}$ and Ag BPEs predominately induced n-doping. This observation demonstrates a proof-of-concept, visual essay to assess the electrode's ability to inject electrons or holes into the luminescent CP. Although each BPE array of this study consisted of the same metal, it is possible to fabricate BPE arrays consisted of different conductors for fast, parallel and visual screening of effective metal electrodes.

The images and results of Figure 4 provide a beautiful demonstration of BPEC. In Figure 4, the bipolar redox reactions are electrochemical doping reactions visualized as PL-quenched regions atop and below the BPEs. The pand $n$-doped areas of the thirteen BPEs varied greatly, but the $n / p$ area ratios are highly consistent. The constant $n / p$ ratio indicates the redox reactions induced by the BPE are coupled. For the BPE to remain electrically neutral, the redox reactions must be balanced. Hence, a constant area ratio is an indication that the electrochemical $p$ - and ndoping the luminescent CP are balanced. Based on the area ratios, we can deduce that the level of doping (dopant charge per CP unit) of the p-doped region is approximately half that of the $n$-doped region.

The large spread in $\mathrm{n} / \mathrm{p}$ area ratio during the initial stage of operation, on the other hand, suggests possible chemical involvement of the BPEs and/or the electrolyte on the poles where the expected reactions are lacking. In regular PLECs made with Au DEs without any BPEs, the delayed onset of n-doping on the negative DE has been attributed to undesired side-reactions involving the electrolyte.58 Meanwhile, PLECs made with Al DEs can experience significant delay in the onset of p-doping when the operating voltage is insufficient.59 In general, the work function of $\mathrm{DE}$ has been shown to strongly affect the doping initiation in regular PLECs.60 In this study, we have shown that the same sensitivity also exists when the metals are formed as wireless BPEs between the wired
DEs. This is the basis of the BPE-based screening for metal electrodes.

An amazing observation is the results of Figure 5. The highly divergent $n / p$ area ratios converge into a single value as the doped area increases. This unexpected phenomenon has a simple explanation. Initially, the BPEs are pure, evaporated metal discs. The redox doping reactions are induced at the metal/polymer interfaces when the DEs are biased. As soon as the CP is doped on one of poles, the reaction (doping) front shifts to the interface between the doped and undoped CP. When this happens, the BPE now constitutes the original metal disc as well as the doped, conductive CP. The new, enlarged BPE facilitates the doping reaction on the other metallic pole due to an increased potential drop at the extremity of the new, composite BPE. This is partly responsible for the accelerated growth of the doped areas. Subsequently, the BPE encompasses the $\mathrm{p}$ - and $\mathrm{n}$-doped regions interconnected by the original metal disc BPE. The new, composite BPE is dynamic in size and composition. When the doped $\mathrm{CP}$ has expanded to a sufficient size, the composite BPE losses the characteristics of the original metal BPE. The relative rate of redox doping reactions, as measured by the $n / p$ area ratio, is now entirely determined by the mixed SPE/CP itself under the specific operating voltage and temperature. This realization suggests that the BPE arrays can not only be used to assess the BPE metals, but also the SPE/CP blend itself. The fact that the converged area ratios are different at $360 \mathrm{~K}$ and $340 \mathrm{~K}$ support this assertion as we know the mobility of electrolyte ions are strongly temperature dependent. Research is currently underway to explore other factors that may affect the final $n / p$ area ratio.

Finally, we note that the BPE doping do retain some distinct features even after the $n / p$ area ratio has converged. For example, the BPE doping from the Al BPEs has a much slender profile than that of Ag and Au BPEs, as shown in Figure S2. In Figure 4, the BPE doping from the out-most BPEs (\#1 and \#13) are tilted and the largest in size. This is not caused by any variation or distortion of the BPEs. Rather, it is caused by a local electric field which is oriented in the direction of doping and is stronger than the electric field near the inner BPEs. This fringe effect becomes stronger as the BPE grows in size. A COMSOL simulation, shown in Figure S3, demonstrates the variation in electric field strength and direction near the BPE array.

\section{CONCLUSION}

In this work, we demonstrated extremely large planar PLECs with a horizontal array of metallic BPEs made of Ag, $\mathrm{Al}$ and $\mathrm{Au}$. This simple device structure was used to visually assess the ability of metals to inject charges into a luminescent polymer. When the PLEC is polarized, the individual discs of the BPE array induced highly visible electrochemical $\mathrm{p}$ - and $\mathrm{n}$-doping within the luminescent CP. The three BPE arrays initially generated distinct doping patterns that are consistent with their work function differences. Over time, the doped regions grew to a size that is much larger than that of the original metal BPE. When this happened, the $n / p$ area ratio of all BPEs, 
regardless of the metal type, converged to a single value. Based on this observation, we conclude that the $\mathrm{p}$ - and ndoped CPs, joined by the original metal BPE, have become a new, dynamic BPE whose ability to induce additional doping is no longer affected by the BPE metal. The internal structure of the dynamic BE, as measured by the $n / p$ area ratio, reflects only the properties of the mixed conductor of the PLEC active layer itself when it reaches certain size.

\section{SUPPORTING INFORMATION}

Figure S1 Initial doping patterns of $\mathrm{Al}, \mathrm{Ag}$ and $\mathrm{Au} \mathrm{BPE}$ arrays tested at $340 \mathrm{~K}$.

Figure S2 Doping patterns of all six BPE arrays after the BPE doping has significantly increased in size. The $\mathrm{p}$ - and n-doped regions display near mirror symmetry in size for all 78 BPEs.

Figure S3 COMSOL simulation of electric field profile around the BPE array, showing variation in electric field strength along the BPE array.

\section{Corresponding Author}

*jungao@queensu.ca

\section{ACKNOWLEDGMENT}

This research was supported by the Natural Sciences and Engineering Research Council of Canada (Grant Number 2015-05344).

\section{ABBREVIATIONS}

BPEC, Bipolar electrochemistry; BPE, bipolar electrode; LEC, light-emitting electrochemical cell; $\mathrm{DE}$, driving electrode; $\mathrm{CP}$, conjugated polymer; SPE, solid polymer electrolyte; PL, photoluminescence; EL, electroluminescence; PLEC, polymer light-emitting electrochemical cell; PEO, polyethylene oxide; KTf, potassium trifluoromethanesulfonate; MEH-PPV, poly[2methoxy-5-(2-ethylhexyloxy)-1,4-phenylenevinylene].

\section{REFERENCES}

1. Pei, Q. B.; Yu, G.; Zhang, C.; Yang, Y.; Heeger, A. J., Polymer Light-Emitting Electrochemical-Cells. Science 1995, 269 (5227), 10861088.

2. Gao, J., Polymer light-emitting electrochemical cells-Recent advances and future trends. Current Opinion in Electrochemistry 2018, 7, 87-94.

3. Fresta, E.; Costa, R. D., Beyond traditional light-emitting electrochemical cells - a review of new device designs and emitters. Journal of Materials Chemistry C 2017, 5 (23), 5643-5675.

4. Tang, S.; Edman, L., Light-Emitting Electrochemical Cells: A Review on Recent Progress. Topics in Current Chemistry 2016, 374 (4).

5. Meier, S. B.; Tordera, D.; Pertegas, A.; Roldan-Carmona, C.; Orti, E.; Bolink, H. J., Light-emitting electrochemical cells: recent progress and future prospects. Materials Today 2014, 17 (5), 217-223.

6. Weber, M. D.; Fresta, E.; Elie, M.; Miehlich, M. E.; Renaud, J. L.; Meyer, K.; Gaillard, S.; Costa, R. D., Rationalizing Fabrication and Design Toward Highly Efficient and Stable Blue Light-Emitting Electrochemical Cells Based on NHC Copper(I) Complexes. Advanced Functional Materials 2018, 28 (17).

7. Su, H. C., Optical Techniques for Light-Emitting Electrochemical Cells. Chempluschem 2018, 83 (4), 197-210.

$8 . \quad$ Nishikitani, Y.; Cho, T.; Uchida, S.; Nishimura, S.; Oyaizu, K.; Nishide, H., Polymer-Based White-Light-Emitting Electrochemical Cells with Very High Color-Rendering Index Based on Blue-Green Fluorescent
Polyfluorenes and Red-Phosphorescent Iridium Complexes. Chempluschem 2018, 83 (5), 463-469.

9. $\quad$ Nagatsu, G.; Sakanoue, T.; Tane, S.; Yonekawa, F.; Takenobu, T., An ester-substituted polyfluorene derivative for light-emitting electrochemical cells: bright blue emission and its application in a hostguest system. Materials Chemistry Frontiers 2018, 2 (5), 952-958.

$10 . \quad$ Moore, M. D.; Bowler, M. H.; Reynolds, J. E.; Lynch, V. M.; Shen, Y. L.; Slinker, J. D.; Sessler, J. L., lonic Organic Small Molecules as Hosts for Light-Emitting Electrochemical Cells. Acs Applied Materials \& Interfaces 2018, 10 (29), 24699-24707.

11. Jenatsch, S.; Regnat, M.; Hany, R.; Diethelm, M.; Nuesch, F.; Ruhstaller, B., Time-Dependent $\mathbf{p - i - n}$ Structure and Emission Zone in Sandwich-Type Light-Emitting Electrochemical Cells. Acs Photonics 2018, 5 (4), 1591-1598.

12. He, L.; Wang, X. X.; Duan, L., Enhancing the Overall Performances of Blue Light-Emitting Electrochemical Cells by Using an Electron-Injecting/Transporting lonic Additive. Acs Applied Materials \& Interfaces 2018, 10 (14), 11801-11809.

13. Gao, J.; Dane, J., Planar polymer light-emitting electrochemical cells with extremely large interelectrode spacing. Applied Physics Letters 2003, 83 (15), 3027-3029.

14. Hu, Y.; Gao, J., Direct Probing of a Polymer Electrolyte/Luminescent Conjugated Polymer Mixed lonic/Electronic Conductor. Journal of the American Chemical Society 2009, 131 (51), 18236-18237.

15. Pei, Q.; Yang, Y.; Yu, G.; Zhang, C.; Heeger, A. J., Polymer LightEmitting Electrochemical Cells: In Situ Formation of a Light-Emitting p-n Junction. Journal of the American Chemical Society 1996, 118 (16), 39223929.

16. Matyba, P.; Maturova, K.; Kemerink, M.; Robinson, N. D.; Edman, L., The dynamic organic $p-n$ junction. Nature Materials 2009, 8 (8), 672676.

17. Hu, Y.; Gao, J., Direct Imaging and Probing of the $p-n$ Junction in a Planar Polymer Light-Emitting Electrochemical Cell. Journal of the American Chemical Society 2011, 133 (7), 2227-2231.

18. AlTal, F.; Gao, J., High resolution scanning optical imaging of a frozen polymer p-n junction. Journal of Applied Physics 2016, 120 (11), 115501.

19. Tang, S.; Tan, W.-Y.; Zhu, X.-H.; Edman, L., Small-molecule light-emitting electrochemical cells: evidence for in situ electrochemical doping and functional operation. Chemical Communications 2013, 49 (43), 4926-4928.

20. Meier, S. B.; van Reenen, S.; Lefevre, B.; Hartmann, D.; Bolink, H. J.; Winnacker, A.; Sarfert, W.; Kemerink, M., Dynamic Doping in Planar lonic Transition Metal Complex-Based Light-Emitting Electrochemical Cells. Advanced Functional Materials 2013, 23 (28), 3531-3538.

21. Chen, S.; Wantz, G.; Bouffier, L.; Gao, J., Solid-State Bipolar Electrochemistry: Polymer-Based Light-Emitting Electrochemical Cells. ChemElectroChem 2016, 3 (3), 392-398.

22. Fattah, Z.; Loget, G.; Lapeyre, V.; Garrigue, P.; Warakulwit, C.; Limtrakul, J.; Bouffier, L.; Kuhn, A., Straightforward single-step generation of microswimmers by bipolar electrochemistry. Electrochimica Acta 2011, 56 (28), 10562-10566.

23. Jiang, J. Z.; Guo, M. H.; Yao, F. Z.; Li, J.; Sun, J. J., Propulsion of copper microswimmers in folded fluid channels by bipolar electrochemistry. Rsc Advances 2017, 7 (11), 6297-6302.

$24 . \quad$ Sentic, M.; Arbault, S.; Bouffier, L.; Manojlovic, D.; Kuhn, A.; Sojic, N., 3D electrogenerated chemiluminescence: from surface-confined reactions to bulk emission. Chem. Sci. 2015, 6 (8), 4433-4437.

25. Ishiguro, Y.; Inagi, S.; Fuchigami, T., Gradient Doping of Conducting Polymer Films by Means of Bipolar Electrochemistry. Langmuir 2011, 27 (11), 7158-7162.

26. Lundgren, A.; Munktell, S.; Lacey, M.; Berglin, M.; Bjorefors, F., Formation of Gold Nanoparticle Size and Density Gradients via Bipolar Electrochemistry. ChemElectroChem 2016, 3 (3), 378-382.

27. Ramakrishnan, S.; Shannon, C., Display of Solid-State Materials Using Bipolar Electrochemistry. Langmuir 2010, 26 (7), 4602-4606.

28. Tan, S. M.; Pumera, M., Composition-Graded MoWSx Hybrids with Tailored Catalytic Activity by Bipolar Electrochemistry. Acs Applied Materials d Interfaces 2017, 9 (48), 41955-41964.

29. Inagi, S., Fabrication of gradient polymer surfaces using bipolar electrochemistry. Polymer Journal 2016, 48 (1), 39-44. 
30. Lotfi, S.; Navaee, A.; Salimi, A., Light-Driven Photocatalytic Hydrogen Evolution on Spindle-like MoSx Nanostructures Grown on PolySalicylic Acid Synthesized through Bipolar Electrochemistry. Acs Sustainable Chemistry \& Engineering 2018, 6 (8), 9784-9792.

31. Ongaro, M.; Gambirasi, A.; Ugo, P., Closed Bipolar Electrochemistry for the Low-Potential Asymmetrical Functionalization of Micro- and Nanowires. ChemElectroChem 2016, 3 (3) , 450-456.

32. Gupta, B.; Goudeau, B.; Kuhn, A., Wireless Electrochemical Actuation of Conducting Polymers. Angewandte Chemie-International Edition 2017, 56 (45), 14183-14186.

33. Loget, G.; Zigah, D.; Bouffier, L.; Sojic, N.; Kuhn, A., Bipolar Electrochemistry: From Materials Science to Motion and Beyond. Accounts of Chemical Research 2013, 46 (11), 2513-2523.

34. Fosdick, S. E.; Knust, K. N.; Scida, K.; Crooks, R. M., Bipolar Electrochemistry. Angewandte Chemie-International Edition 2013, 52 (40), 10438-10456.

35. Koefoed, L.; Pedersen, S. U.; Daasbjerg, K., Bipolar electrochemistry-A wireless approach for electrode reactions. Current Opinion in Electrochemistry 2017, 2 (1), 13-17.

36. Gao, J.; Chen, S.; AlTal, F.; Hu, S.; Wantz, G.; Bouffier, L., Bipolar Electrode Array Embedded in a Polymer Light-Emitting Electrochemical Cell. Acs Applied Materials \& Interfaces 2017, 9 (37), 32405-32410.

37. Hu, S. Y.; Chi, X.; Chen, S. L.; AITal, F.; Gao, J., Visualizing the Bipolar Electrochemistry of Electrochemically Doped Luminescent Conjugated Polymers. Journal of Physical Chemistry C 2017, 121 (15) 8409-8415.

38. Hu, S. Y.; Gao, J., Wireless Electroluminescence: Polymer LightEmitting Electrochemical Cells with Inkjet-Printed 1D and 2D Bipolar Electrode Arrays. Journal of Physical Chemistry C 2018, 122 (16), 9054 9061.

39. AITal, F.; Gao, J., Laser-Induced Bipolar Electrochemistry-OnDemand Formation of Bipolar Electrodes in a Solid Polymer Light-Emitting Electrochemical Cell. Journal of the American Chemical Society 2018, 140 (30), 9737-9742.

40. $\quad$ Tracy, C.; Gao, J., Polymer Bulk Homojunction Photonic Devices. Applied Physics Letters 2005, 87, 143502.

41. Tracy, C.; Gao, J., Polymer bulk homojunction light-emitting electrochemical cells. Journal of Applied Physics 2006, 100, 104503.

42. Bonnet, W.; Tracy, C.; Wantz, G.; Liu, G. J.; Gao, J., Bulk Electroluminescence from Conjugated Polymer Thin Films via the Formation of Gold Nanoislands. Small 2008, 4 (10), 1707-1710.

43. Zhang, X. W.; Zhang, L. L.; Zhai, Q. F.; Gu, W. L.; Li, J.; Wang, E. K., Self-Powered Bipolar Electrochromic Electrode Arrays for Direct Displaying Applications. Anal. Chem. 2016, 88 (5), 2543-2547.

44. Koefoed, L.; Pedersen, E. B.; Thyssen, L.; Vinther, J.; Kristiansen, T.; Pedersen, S. U.; Daasbjerg, K., Functionalizing Arrays of Transferred Monolayer Graphene on Insulating Surfaces by Bipolar Electrochemistry. Langmuir 2016, 32 (25), 6289-6296.

45. Wu, M. S.; Liu, Z.; Shi, H. W.; Chen, H. Y.; Xu, J. J., Visual Electrochemiluminescence Detection of Cancer Biomarkers on a Closed Bipolar Electrode Array Chip. Anal. Chem. 2015, 87 (1), 530-537.

46. Zhang, J. D.; Yu, T.; Li, J. Y.; Xu, J. J.; Chen, H. Y., An ITO bipolar array for electrochemiluminescence imaging of H202. Electrochem. Commun. 2014, 49, 75-78.

47. Chang, B.-Y.; Chow, K.-F.; Crooks, J. A.; Mavre, F.; Crooks, R. M., Two-channel microelectrochemical bipolar electrode sensor array. Analyst 2012, 137 (12), 2827-2833.

48. Xiao, Y.; Xu, L. R.; Qi, L. W., Electrochemiluminescence bipolar electrode array for the multiplexed detection of glucose, lactate and choline based on a versatile enzymatic approach. Talanta 2017, 165, 577-583.

49. Khoshfetrat, S. M.; Ranjbari, M.; Shayan, M.; Mehrgardi, M. A.; Kiani, A., Wireless Electrochemiluminescence Bipolar Electrode Array for Visualized Genotyping of Single Nucleotide Polymorphism. Anal. Chem. 2015, 87 (16), 8123-8131.

50. Zhai, Q. F.; Zhang, X. W.; Han, Y. C.; Zhai, J. F.; Li, J.; Wang, E. K., A Nanoscale Multichannel Closed Bipolar Electrode Array for Electrochemiluminescence Sensing Platform. Anal. Chem. 2016, 88 (1), 945-951.

51. Termebaf, H.; Shayan, M.; Kiani, A., Two-Step Bipola Electrochemistry: Generation of Composition Gradient and Visual Screening of Electrocatalytic Activity. Langmuir 2015, 31 (48), 13238-13246.

52. Lin, X.; Zheng, L.; Gao, G.; Chi, Y.; Chen, G., Electrochemiluminescence Imaging-Based High-Throughput Screening
Platform for Electrocatalysts Used in Fuel Cells. Anal. Chem. 2012, 84 (18) 7700-7707

53. Fosdick, S. E.; Berglund, S. P.; Mullins, C. B.; Crooks, R. M., Parallel Screening of Electrocatalyst Candidates Using Bipolar Electrochemistry. Anal. Chem. 2013, 85 (4), 2493-2499.

54. Munktell, S.; Nyholm, L.; Bjorefors, F., Towards high throughput corrosion screening using arrays of bipolar electrodes. Journal of Electroanalytical Chemistry 2015, 747, 77-82.

55. Yuan, F.; Qi, L.; Fereja, T. H.; Snizhko, D. V.; Liu, Z.; Zhang, W.; $\mathrm{Xu}, \mathrm{G}$., Regenerable bipolar electrochemiluminescence device using glassy carbon bipolar electrode, stainless steel driving electrode and cold patch. Electrochimica Acta 2018, 262, 182-186.

56. Zhou, M.; Png, R. Q.; Khong, S. H.; Sivaramakrishnan, S.; Zhao, L. H.; Chua, L. L.; Friend, R. H.; Ho, P. K. H., Effective work functions for the evaporated metal/organic semiconductor contacts from in-situ diode flatband potential measurements. Applied Physics Letters 2012, 101 (1).

57. Parker, I. D., Carrier Tunneling and Device Characteristics in Polymer Light-Emitting-Diodes. Journal of Applied Physics 1994, 75 (3), 1656- 1666.

58. Fang, J.; Matyba, P.; Robinson, N. D.; Edman, L., Identifying and alleviating electrochemical side-reactions in light-emitting electrochemical cells. Journal of the American Chemical Society 2008, 130 (13), 4562 4568.

59. Shin, J. H.; Matyba, P.; Robinson, N. D.; Edman, L., The influence of electrodes on the performance of light-emitting electrochemical cells. Electrochimica Acta 2007, 52 (23), 6456-6462.

60. Hohertz, D.; Gao, J., How electrode work function affects doping and electroluminescence of polymer light-emitting electrochemical cells. Advanced Materials 2008, 20 (17), 3298-3302. 